\section{Leishmaniose visceral e sua relação com fatores climáticos e ambientais no Estado do Tocantins, Brasil, 2007 a 2014}

\author{
Visceral leishmaniasis and its relationship with \\ climate and environmental factors in the State of \\ Tocantins, Brazil, from 2007 to 2014
}

\section{Leishmaniasis visceral y su relación con factores climáticos y ambientales en el Estado de Tocantins, Brasil, 2007 a 2014}

Lisiane Lappe dos Reis 1 Antônio Alcirley da Silva Balieiro 1 Fernanda Rodrigues Fonseca 1 Maria Jacirema Ferreira Gonçalves 1,2

doi: 10.1590/0102-311X00047018

\section{Resumo}

A leishmaniose visceral ( $L V)$ pode ser influenciada por fatores climáticos e ambientais. Tocantins é o estado com mais incidência no Brasil, contribuindo para o aumento dos casos na macrorregião Norte. Este estudo tem objetivo de identificar a correlação entre a taxa de incidência de LV e fatores climáticos e ambientais, nos municípios do Estado do Tocantins de 2007 a 2014. As correlações entre a taxa de incidência bruta de LV e as variáveis elevação, precipitação, Índice de Vegetação Melhorado (EVI - Enhanced Vegetation Index) e temperatura foram testadas por meio dos indices de Moran Global e Local, identificando também clusters de incidência de $L V$. A análise de variância para tendência linear foi testada entre as médias das variáveis em cada categoria da taxa. Os resultados apontam aumento nas taxas de incidência, na medida em que aumentam os valores de precipitação anual, umidade, EVI e temperatura noturna; e uma relação inversa para a elevação e temperatura diurna. Os clusters identificados evidenciam que variáveis climáticas e ambientais se relacionam à incidência de $L V$, devendo haver outros estudos que ajustem os fatores associados aos sujeitos, a fim de dimensionar o quanto o ambiente ou a influência do homem nele impacta na doença.

Temperatura Ambiente; Pluviometria; Altitude; Umidade;

Leishmaniose Visceral

\author{
Correspondência \\ L. L. Reis \\ Instituto Leônidas es Maria Deane, Fundação Oswaldo Cruz. \\ Rua Teresina 476, Manaus, AM 69065-360, Brasil. \\ lisiane_reis@icloud.com \\ 1 Instituto Leônidas es Maria Deane, Fundação Oswaldo Cruz, \\ Manaus, Brasil. \\ 2 Escola de Enfermagem de Manaus, Universidade Federal do \\ Amazonas, Manaus, Brasil.
}




\section{Introdução}

A leishmaniose visceral (LV) é uma doença causada pelo protozoário intracelular Leishmania infantum e transmitida por vetores, especialmente o flebotomíneo Lutzomyia longipalpis, cuja relação com fatores climáticos, ambientais e sociais tem sido explorada. No entanto, ainda se buscam explicações que permitam compreender a doença que, no Estado do Tocantins, apresenta a mais elevada incidência do Brasil (taxa de incidência 26,2/100 mil habitantes no período de 2007 a 2014) 1

Ao longo do tempo, a Região Nordeste apresentava a maior incidência de LV no Brasil, particularmente nos estados de Piauí e Maranhão. Porém, no período deste estudo, 2007 a 2014, a Região Norte ultrapassou a Nordeste em incidência de LV, devido à elevada incidência no Estado de Tocantins.

Já foi apontada a correlação positiva entre a incidência de LV com áreas de tensão ecológica, presença de floresta ombrófila e área urbana no Estado do Tocantins, assim como correlação negativa entre LV, floresta de savana e cultura agrícola 2 . Nesta proposta, acrescentam-se outros fatores como: elevação, precipitação pluviométrica, umidade, Índice de Vegetação Melhorado (Enhanced Vegetation Index - EVI) e temperatura.

A carência de estudos explicando essa situação da LV em Tocantins leva ao questionamento se os fatores climáticos e ambientais se correlacionam à ocorrência de LV neste estado, que apresenta características do bioma amazônico e de seu limite com o cerrado ${ }^{3}$. Uma ferramenta que contribui para essa abordagem é a utilização de técnicas de análise espacial, que permitem estudar a aleatoriedade espacial de dados, evidenciando dependência espacial e correlação entre ambiente e LV.

O objetivo do presente estudo foi identificar a correlação entre a taxa de incidência de LV e fatores climáticos e ambientais nos municípios do Estado do Tocantins, de 2007 a 2014.

\section{Métodos}

\section{Considerações éticas}

Estudo realizado com todos os casos novos de LV humana, doença de notificação compulsória 4, notificados ao Sistema de Informação de Agravos de Notificação (SINAN). Os casos novos notificados foram obtidos do Ministério da Saúde em 14 de setembro de 2015, referentes ao período de 2007 a 2014, sem a identificação dos sujeitos e já excluídas as duplicidades. Portanto, há dispensa de avaliação pelo Comitê de Ética em Pesquisa, conforme a Resolução no 510/2016 do Conselho Nacional de Saúde 5.

\section{Desenho de estudo}

Estudo ecológico e analítico que utiliza como unidades de análise os municípios do Estado do Tocantins.

Foram incluídos os casos novos notificados de LV e analisados de acordo com o município de residência. Os casos excluídos foram: recaídas da doença e casos de LV transferidos ou não confirmados.

\section{Área de estudo}

Tocantins tem um território de $277.720 .412 \mathrm{~km}^{2}$ e possuía 1.383 .453 habitantes em 2010 (Departamento de Informática do SUS. http://www2.datasus.gov.br, acessado em 03/Out/2016). O estado se localiza na área central do país e é formado por 139 municípios e, dentre eles, 112 notificaram casos de LV no período de 2007 a 2014.

\section{Taxa de incidência de LV}

A taxa de incidência acumulada do período de 2007 a 2014 foi calculada para cada município do Estado de Tocantins, utilizando-se o somatório dos casos novos notificados no período como numerador, assim como o somatório da população de cada município do período como denominador, multiplicando-se por 100 mil habitantes. Os dados populacionais foram obtidos do Departamento 
de Informática do SUS (DATASUS; http://www2.datasus.gov.br, acessado em 03/Out/2016), em que já estão disponíveis dados de estimativas, censo demográfico e projeções populacionais, de acordo com o Instituto Brasileiro de Geografia e Estatística (IBGE; http://www.ibge.gov.br/estadosat/perfil. php? sigla=to, acessado em 03/Jul/2017).

A categorização das taxas de incidência obedece aos pontos de corte utilizados pela Organização Pan-Americana da Saúde (OPAS) 6, entretanto as taxas das categorias muito intensa e intensa foram agrupadas com as taxas na categoria alta. Os municípios que não apresentaram notificação no período foram classificados como "sem casos". Desse modo, utilizam-se quatro categorias de taxas (casos por 100 mil habitantes): baixa $(0,01 \mid--9,55)$, média $(9,55 \mid--23,04)$ e alta $(\geq 23,04)$, e sem casos.

\section{Taxa de urbanização}

A taxa de urbanização foi calculada utilizando-se o quantitativo da população urbana dos municípios do Estado de Tocantins como numerador e a população total dos municípios como denominador, multiplicando-se por 100. A taxa de urbanização representa o percentual de população da área urbana em relação à população total (IBGE; https://censo2010.ibge.gov.br/sinopse/index.php?uf=17\&da dos $=8$, acessado em 28/Fev/2018), que segundo definição do IBGE 7 refere-se à concentração de população em áreas urbanas.

\section{Dados ambientais}

As variáveis climáticas e ambientais, em nível municipal, que foram obtidas na plataforma do Google Earth Engine (https://code.earthengine.google.com/, acessado em 08/Mar/2017), são: elevação, precipitação pluviométrica média anual, umidade do ar específica mínima e máxima, temperatura média diurna e noturna do ano de 2010, e EVI dos anos de 2007, 2010 e 2014. A elevação foi extraída do Shuttle Radar Topography Mission (SRTM) 8 em uma resolução de 3 arco-segundo (aproximadamente 90m). A precipitação pluviométrica foi extraída do Climate Hazards Group InfraRed Precipitation with Station Data (CHIRPS) 9 como precipitação média anual por município em mm. A umidade do ar específica mínima e máxima $(\mathrm{g} / \mathrm{kg})$ a $2 \mathrm{~m}$ de altura do solo com medição em intervalos de 6 horas foi extraída do modelo de previsão do tempo Climate Forecast System (CFS) do Centro Nacional de Previsão Ambiental dos Estados Unidos (NCEP) 10. As temperaturas médias noturna e diurna foram extraídas do Land Surface Temperature 11 LST_Day_ $1 \mathrm{~km}$ e LST_Night_1 $1 \mathrm{~km}$ : temperatura diurna e noturna da superfície terrestre $(\mathrm{K})$, transformadas para ${ }^{\circ} \mathrm{C}$. Os dados de EVI 12 foram extraídos do satélite MOD13Q1 pelas bandas Near-IR, Red e Blue, com resolução de 250m e composição de 16 em 16 dias. Os dados de EVI são transformações espectrais de três bandas do satélite projetadas para obter informações da vegetação pela atividade fotossintética terrestre e variações estruturais do dossel ${ }^{12}$. Como o EVI varia pouco ao longo do tempo, os dados foram coletados de três momentos, que representam o período do estudo: início (2007), meio (2010) e final (2014).

\section{Análise estatística}

A taxa de incidência de LV juntamente com as variáveis taxa de urbanização, elevação, precipitação, umidade mínima e máxima, EVI 2007, EVI 2010, EVI 2014 e temperatura noturna e diurna foram exploradas descritivamente, observando a distribuição da média e desvio padrão conforme as categorias da taxa.

A associação entre as médias das variáveis em cada categoria de taxa foi testada pela análise de variância (ANOVA) para tendência linear e o valor de $\mathrm{p}$ foi avaliado pelo teste $\mathrm{F}$, considerando-se a significância estatística menor que $1 \%$. Previamente, em todas as variáveis foi realizado o teste de normalidade, por meio do teste de Shapiro-Wilk. As análises foram realizadas no software Stata, versão 13 (https://www.stata.com).

As seguintes variáveis foram plotadas em gráficos de distribuição do tipo box plot, a fim de ilustrar a tendência linear, conforme cada categoria da taxa de incidência de LV: elevação, temperatura diurna e noturna, EVI de 2007 e 2014 e precipitação pluviométrica. 
A urbanização foi excluída dos gráficos box plot e da análise espacial, por não ser estatisticamente significativa na ANOVA. Referente aos dados ambientais, optou-se por excluir dos gráficos box plot e do mapeamento de cluster as seguintes variáveis: EVI 2010, por apresentar resultado semelhante ao EVI 2014; e umidade máxima e mínima, pois apresentaram os menores Índices de Moran (I de Moran) Global bivariado.

\section{- Autocorrelação espacial Global e Local bivariada}

O I de Moran Global bivariado foi calculado para verificar a correlação entre uma variável em um município e uma variável diferente nos municípios vizinhos que pertencem a toda área do estudo. Essa estatística fornece uma indicação do grau de associação linear (positiva ou negativa) entre o valor de uma variável em um local e a média de outra variável em locais vizinhos. A hipótese nula é de independência espacial, neste caso seu valor seria zero. A estatística pode variar de $-1 \mathrm{a}+1$. Valores positivos (entre 0 e +1 ) indicam para correlação direta e, valores negativos (entre 0 e -1 ) correlação inversa 13,14 .

Como o I de Moran Global fornece um valor único, como medida de associação espacial para todo o conjunto de dados, não demonstrando padrões locais de associação espacial, foi calculado o I de Moran Local bivariado. Nessa técnica, somente os municípios definidos em uma matriz de vizinhança espacial são incluídos no cálculo. Tal índice fornece o grau de autocorrelação espacial estatisticamente significativo, em cada unidade regional. Uma maior similaridade dos dados, que a de aleatoriedade espacial, sugere um agrupamento espacialmente similar com as duas variáveis. Uma dissimilaridade maior do que a da aleatoriedade espacial implicaria uma relação forte, local e negativa entre as duas variáveis. A análise local é visualizada na forma de mapas de clusters de Indicador Local de Associação Espacial (LISA; Local Indicators of Spatial Association). Para a análise de correlação espacial local bivariada, foi utilizada a matriz de contiguidade Queen de ordem 1 calculada no software GeoDa ${ }^{\circledR}$, versão 1.12 (https://spatial.uchicago.edu/software), que considera vizinhas as regiões que apresentem fronteiras comuns.

Utilizou-se o pseudo valor de p com 999 permutações para avaliar a significância de 1\%, tanto para o I de Moran Global, quanto para o I de Moran Local 14.

\section{Resultados}

Foram notificados 2.885 casos de LV em Tocantins no período de 2007 a 2014, cuja taxa de incidência foi 26,2/100 mil habitantes, e média anual de 360,6 casos $\pm 105,9$. A menor taxa foi registrada em 2014 (11,16/100 mil habitantes) e a maior em 2011 (34,69/100 mil habitantes). Quanto às variáveis ambientais, seus valores mínimos e máximos são os seguintes:

- Elevação média (m): 102,1 a 631,6;

- Temperatura diurna média anual $\left({ }^{\circ} \mathrm{C}\right): 28,5$ a 35,3 ;

- Temperatura noturna média anual $\left({ }^{\circ} \mathrm{C}\right): 19,9$ a 22,7 ;

- $\quad$ EVI 2007: 0,25 a 0,49;

- $\quad$ EVI 2010: 0,25 a 0,47;

- EVI 2014: 0,26 a 0,46;

- Precipitação pluviométrica média anual (mm): 1.154,4 a 1.710,2;

- Umidade específica do ar mínima (g/kg): 8,42 a 14,73;

- Umidade específica do ar máxima (g/kg): 10,13 a 16,72.

A distribuição da média das variáveis em relação às categorias da taxa de incidência de LV apresentou associação significativa com todas as variáveis ambientais, com exceção da urbanização (Tabela 1). Todas as variáveis testadas apresentaram tendência linear positiva significativa, as quais aumentaram à medida que a taxa de incidência de LV aumenta, com exceção da elevação e temperatura média diurna que apresentaram correlação negativa as quais diminuem conforme aumento das categorias de taxa (Figura 1). Essa associação também foi testada pelo I de Moran Global bivariado, com resultados estatisticamente significativos (pseudo valor de $\mathrm{p}<0,01$ ). Portanto, a hipótese nula de aleatoriedade espacial foi rejeitada. A variável que teve mais correlação positiva com a taxa de LV foi o EVI 2007 


\section{Tabela 1}

Medidas sumárias da urbanização, elevação, temperatura média diurna e noturna, umidade do ar específica máxima e mínima, Índice de Vegetação Melhorado (EVI - Enhanced Vegetation Index) 2007, 2010 e 2014 e precipitação pluviométrica anual conforme categorias de taxas de leishmaniose visceral (LV). Tocantins, Brasil, 2007 a 2014.

\begin{tabular}{|c|c|c|c|c|c|}
\hline Variável/Categorização da taxa de incidência de LV & Média & DP & Mínimo & Máximo & Valor de $p$ * \\
\hline \multicolumn{6}{|l|}{ Taxa de incidência de LV } \\
\hline 1. Sem notificação & 0,0 & 0,0 & 0,0 & 0,0 & \\
\hline 2. Baixa $(0,01 \mid--9,55)$ & 5,8 & 2,2 & 0,7 & 9,1 & \\
\hline 3. Média $(9,55 \mid--23,04)$ & 17,0 & 4,3 & 9,6 & 22,9 & \\
\hline 4. Alta $(\geq 23,04)$ & 44,9 & 24,1 & 24,1 & 131,1 & \\
\hline Total & 15,6 & 20,0 & 0,0 & 131,1 & \\
\hline \multicolumn{6}{|l|}{ Urbanização (\%) } \\
\hline 1. Sem notificação & 66,7 & 13,8 & 40,0 & 92,7 & 0,17 \\
\hline 2. Baixa $(0,01 \mid--9,55)$ & 62,5 & 16,4 & 21,6 & 97,7 & \\
\hline 3. Média $(9,55 \mid--23,04)$ & 63,5 & 16,8 & 37,6 & 97,1 & \\
\hline 4. Alta $(\geq 23,04)$ & 71,7 & 16,2 & 38,4 & 96,0 & \\
\hline Total & 65,6 & 16,2 & 21,6 & 97,7 & \\
\hline \multicolumn{6}{|l|}{ Elevação (metros) } \\
\hline 1. Sem notificação & 351,4 & 129,8 & 190,1 & 631,6 & $<0,01$ \\
\hline 2. Baixa $(0,01 \mid--9,55)$ & 301,7 & 109,0 & 102,2 & 627,1 & \\
\hline 3. Média $(9,5 \mid--23,04)$ & 268,8 & 84,0 & 122,7 & 534,3 & \\
\hline 4. Alta $(\geq 23,04)$ & 241,2 & 67,3 & 120,8 & 367,5 & \\
\hline Total & 291,3 & 106,9 & 102,2 & 631,6 & \\
\hline \multicolumn{6}{|l|}{ Temperatura média diurna $\left({ }^{\circ} \mathrm{C}\right)$} \\
\hline 1. Sem notificação & 32,4 & 1,2 & 29,4 & 35,3 & $<0,01$ \\
\hline 2. Baixa $(0,01 \mid--9,55)$ & 31,8 & 1,3 & 28,5 & 34,0 & \\
\hline 3. Média $(9,55 \mid--23,04)$ & 31,4 & 1,4 & 28,6 & 34,3 & \\
\hline 4. Alta $(\geq 23,04)$ & 31,3 & 1,2 & 28,7 & 33,8 & \\
\hline Total & 31,7 & 1,3 & 28,5 & 35,3 & \\
\hline \multicolumn{6}{|l|}{ Temperatura média noturna $\left({ }^{\circ} \mathrm{C}\right)$} \\
\hline 1. Sem notificação & 21,2 & 0,5 & 20,3 & 22,2 & $<0,01$ \\
\hline 2. Baixa $(0,01 \mid--9,55)$ & 21,4 & 0,6 & 20,0 & 22,7 & \\
\hline 3. Média $(9,5 \mid--23,04)$ & 21,7 & 0,5 & 20,5 & 22,4 & \\
\hline 4. Alta $(\geq 23,04)$ & 21,8 & 0,4 & 20,9 & 22,4 & \\
\hline Total & 21,5 & 0,5 & 20,0 & 22,7 & \\
\hline \multicolumn{6}{|l|}{ Umidade do ar específica máxima (g/kg) } \\
\hline 1. Sem notificação & 13,6 & 1,5 & 11,2 & 16,0 & 0,007 \\
\hline 2. Baixa $(0,01 \mid--9,55)$ & 13,9 & 1,5 & 10,1 & 16,7 & \\
\hline 3. Média $(9,5 \mid--23,04)$ & 14,4 & 1,4 & 10,8 & 16,3 & \\
\hline 4. Alta $(\geq 23,04)$ & 14,5 & 1,2 & 11,6 & 16,0 & \\
\hline Total & 14,1 & 1,5 & 10,1 & 16,7 & \\
\hline \multicolumn{6}{|l|}{ Umidade do ar específica mínima (g/kg) } \\
\hline 1. Sem notificação & 12,0 & 1,4 & 8,4 & 14,4 & 0,03 \\
\hline 2. Baixa $(0,01 \mid--9,55)$ & 12,4 & 1,3 & 9,1 & 14,7 & \\
\hline 3. Média $(9,55 \mid--23,04)$ & 12,4 & 1,3 & 9,4 & 14,5 & \\
\hline 4. Alta $(\geq 23,04)$ & 12,8 & 1,2 & 9,3 & 14,5 & \\
\hline Total & 12,4 & 1,3 & 8,4 & 14,7 & \\
\hline
\end{tabular}

(continua) 
Tabela 1 (continuação)

\begin{tabular}{|c|c|c|c|c|c|}
\hline Variável/Categorização da taxa de incidência de LV & Média & DP & Mínimo & Máximo & Valor de $p$ * \\
\hline \multicolumn{6}{|l|}{ EVI 2007} \\
\hline 1. Sem notificação & 0,35 & 0,04 & 0,25 & 0,45 & $<0,01$ \\
\hline 2. Baixa $(0,01 \mid--9,55)$ & 0,37 & 0,04 & 0,31 & 0,47 & \\
\hline 3. Média $(9,55 \mid--23,04)$ & 0,40 & 0,06 & 0,30 & 0,49 & \\
\hline 4. Alta $(\geq 23,04)$ & 0,42 & 0,05 & 0,30 & 0,48 & \\
\hline Total & 0,38 & 0,05 & 0,25 & 0,49 & \\
\hline \multicolumn{6}{|l|}{ EVI 2010} \\
\hline 1. Sem notificação & 0,35 & 0,04 & 0,25 & 0,44 & $<0,01$ \\
\hline 2. Baixa $(0,01 \mid--9,55)$ & 0,37 & 0,04 & 0,31 & 0,46 & \\
\hline 3. Média $(9,55 \mid--23,04)$ & 0,39 & 0,05 & 0,30 & 0,47 & \\
\hline 4. Alta $(\geq 23,04)$ & 0,40 & 0,04 & 0,31 & 0,47 & \\
\hline Total & 0,38 & 0,05 & 0,25 & 0,47 & \\
\hline \multicolumn{6}{|l|}{ EVI 2014} \\
\hline 1. Sem notificação & 0,36 & 0,04 & 0,26 & 0,42 & $<0,01$ \\
\hline 2. Baixa $(0,01 \mid--9,55)$ & 0,37 & 0,03 & 0,32 & 0,44 & \\
\hline 3. Média $(9,55 \mid--23,04)$ & 0,39 & 0,04 & 0,31 & 0,46 & \\
\hline 4. Alta $(\geq 23,04)$ & 0,40 & 0,04 & 0,32 & 0,46 & \\
\hline Total & 0,38 & 0,04 & 0,26 & 0,46 & \\
\hline \multicolumn{6}{|l|}{ Precipitação pluviométrica anual (mm) } \\
\hline 1. Sem notificação & $1.401,6$ & 102,1 & $1.224,1$ & $1.593,5$ & $<0,01$ \\
\hline 2. Baixa $(0,01 \mid--9,55)$ & $1.446,3$ & 102,9 & $1.154,4$ & $1.615,2$ & \\
\hline 3. Média $(9,55 \mid--23,04)$ & $1.499,9$ & 100,7 & $1.215,1$ & $1.697,8$ & \\
\hline 4. Alta $(\geq 23,04)$ & $1.531,2$ & 94,0 & $1.161,8$ & $1.710,2$ & \\
\hline Total & $1.467,6$ & 109,7 & $1.154,4$ & $1.710,2$ & \\
\hline
\end{tabular}

DP: desvio padrão.

Nota: as variáveis precipitação pluviométrica anual, temperatura média diurna e noturna e umidade do ar específica máxima e mínima foram extraídas do ano de 2010. A urbanização foi calculada utilizando a população do ano de 2010. A taxa de incidência acumulada de LV foi do período de 2007 a 2014.

* Teste de tendência linear ANOVA ao nível de significância de 1\%.

( $\mathrm{I}=0,4919)$, seguida da precipitação ( $\mathrm{I}=0,4447)$, EVI $2010(\mathrm{I}=0,4437)$, EVI $2014(\mathrm{I}=0,4397)$. As menores correlações foram: temperatura noturna $(I=0,3174)$, umidade máxima $(I=0,2444)$ e umidade mínima $(I=0,1430)$. A elevação $(I=-0,3205)$, seguida da temperatura diurna $(I=-0,3180)$, apresentou correlação negativa.

A distribuição espacial da taxa de incidência de LV é observada na Figura 2 com as taxas de incidência mais altas encontradas mais ao norte do estado.

Nos mapas de cluster LISA bivariado (Figura 3), entre a taxa de incidência de LV e as variáveis ambientais, foram detectados os seguintes clusters:

- Alto-alto: temperatura noturna (Figura 3c), EVI 2007 (Figura 3d), EVI 2014 (Figura 3e) e precipitação (Figura 3f). A correlação foi significativa e positiva;

- Baixo-baixo: elevação (Figura 3a), temperatura noturna (Figura 3c), EVI 2007 (Figura 3d), EVI 2014 (Figura 3e) e precipitação (Figura 3f). A correlação foi significativa e positiva;

- Baixo-alto: elevação (Figura 3a) e temperatura diurna (Figura 3b). A correlação foi significativa e negativa;

- Alto-baixo: temperatura diurna (Figrua 3b), com correlação significativa negativa. 


\section{Figura 1}

Tendência linear entre a taxa de incidência de leishmaniose visceral (LV) e elevação (1a), temperatura média diurna (1b), temperatura média noturna (1c), Índice de Vegetação Melhorado (EVI - Enhanced Vegetation Index) 2007 (1d) e 2014 (1e) e precipitação pluviométrica (1f). Tocantins, Brasil, 2007 a 2014.
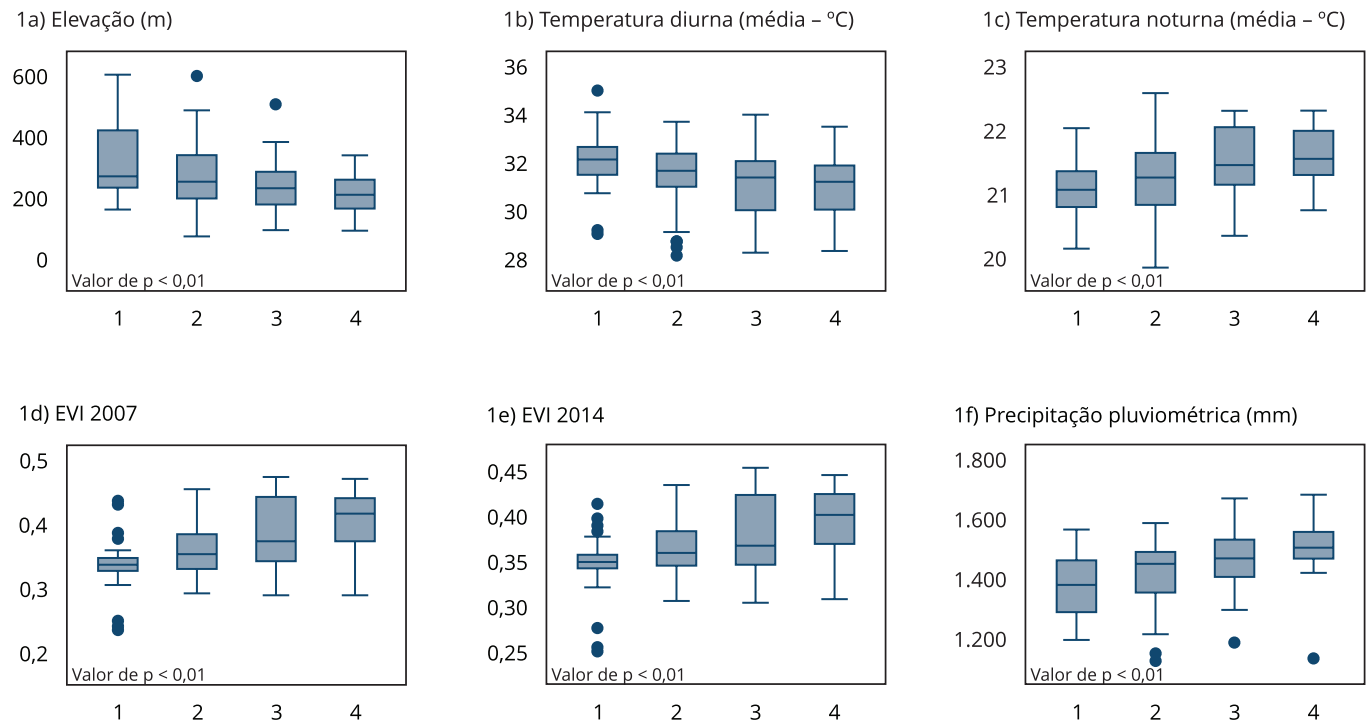

Nota: os números no eixo X indicam categorias da taxa de incidência (2007 a 2014): 1 - sem notificação; 2 - baixa (0,01|--9,55); 3 - média (9,55|--23,04); e 4 - alta ( $\geq 23,04)$. As variáveis ambientais referem-se ao ano de 2010 , com exceção de EVI 2007 e EVI 2014

\section{Discussão}

Este trabalho permitiu identificar que, no Estado do Tocantins, a correlação da taxa de incidência de LV humana com variáveis climáticas e ambientais possui tendência linear significativa. As taxas de incidência aumentam à medida que aumentam os valores de temperatura noturna, umidade do ar máxima e mínima, EVI e precipitação. Por sua vez, elas diminuem com o aumento dos valores médios de temperatura diurna e elevação do terreno. A dependência espacial entre as variáveis ambientais e a taxa de incidência de LV também foi identificada neste trabalho, possibilitando a detecção de clusters que ocorreram, em sua maioria, no Norte e Sul do estado. Isso indica que pode haver condições ambientais propícias à reprodução vetorial e aos reservatórios silvestres da LV, favorecendo o aumento da doença, contudo não se pode descartar o comportamento humano, ou do reservatório canino nesse processo.

O flebotomíneo L. longipalpis na fase larvária se desenvolve em ambientes terrestres úmidos, ricos em matéria orgânica e de baixa incidência luminosa ${ }^{15}$. Por sua vez, os parasitos podem ter o ciclo de vida das formas promastigotas, no vetor, acelerado por pequenos aumentos da temperatura (dentro dos limites de tolerância), aumentando a probabilidade de a fêmea do flebotomíneo sobreviver tempo suficiente para o desenvolvimento do parasito no seu interior. A temperatura ideal para o desenvolvimento do parasito nos vetores é cerca de $25^{\circ} \mathrm{C} 16$. Além disso, o aumento da temperatura também aumenta a densidade de flebotomíneos adultos e, por sua vez, o contato entre vetor e hospedeiros e consequentemente a disseminação da doença 16,17. Corroborando isso, a temperatura pode aumentar a atividade dos flebotomíneos 18 , e repastos sanguíneos repetidos elevam a carga parasitária no vetor 19 , aumentando, dessa forma, a infectividade dos flebotomíneos. 
Mapa de distribuição da taxa de incidência acumulada das notificações de leishmaniose visceral (LV) por 100 mil habitantes nos municípios de Tocantins, Brasil, 2007-2014.
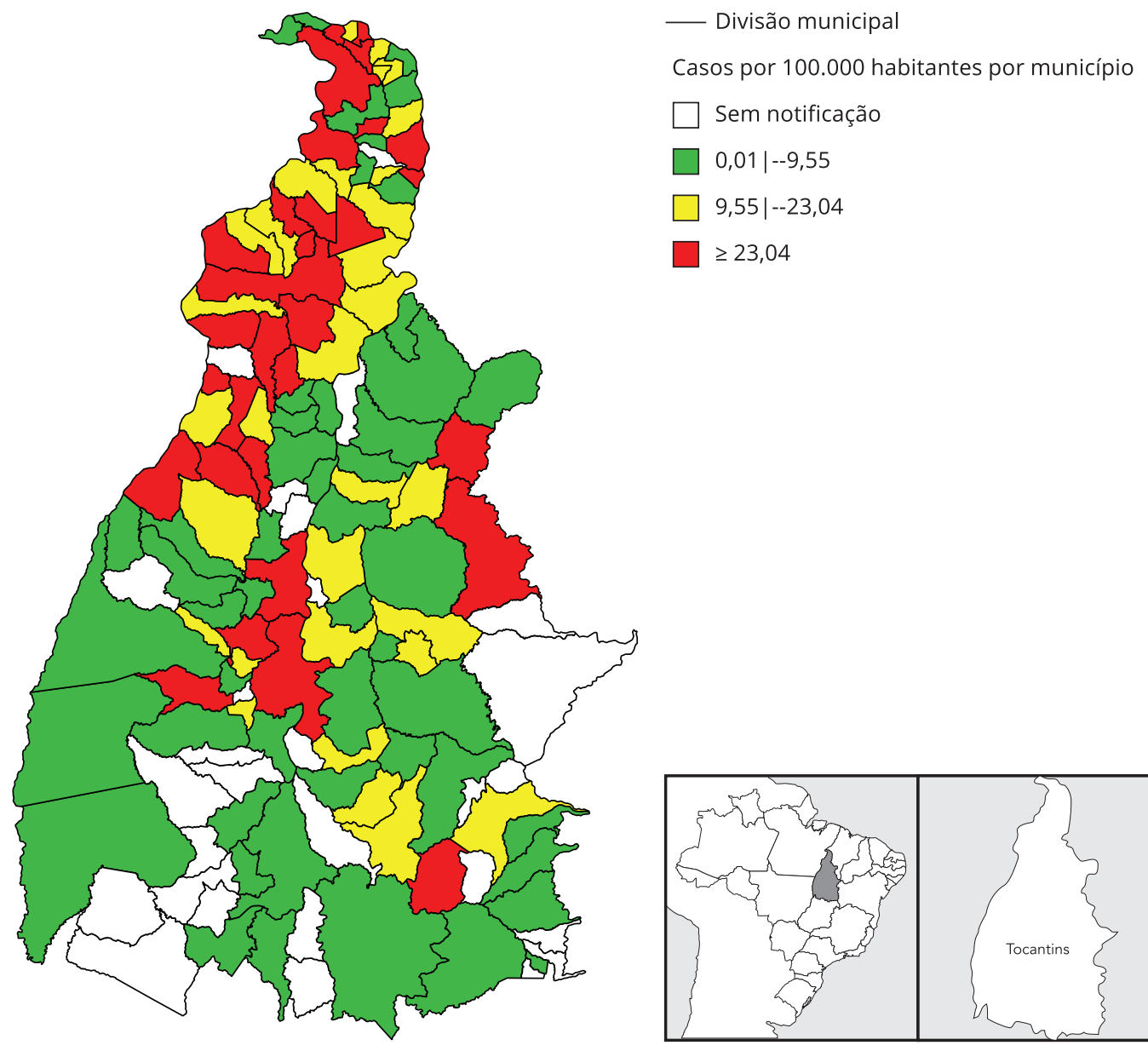

Nota: a categorização das taxas no mapa obedece aos pontos de corte utilizados pela Organização Pan-Americana da Saúde 6, agrupando as taxas intensa e muito intensa, às taxas altas.

Os fatores ligados ao uso do solo, como agricultura, tipo de vegetação e desmatamento, planejamento das cidades e de ocupações humanas necessitam ser considerados quando se pensa em LV. Nesse sentido, poder-se-ia citar a cidade de Araguaína, localizada ao norte do estado, com uma das mais altas taxas de incidência de LV 20, e que, além de possuir fatores climáticos e ambientais favoráveis à doença, sofreu intensa expansão urbana e desmatamento, sem um planejamento e infraestrutura adequados. No entanto, não somente o fator ambiental está relacionado à incidência de LV, mas os fatores associados aos sujeitos, como, por exemplo, a imunidade, estado nutricional, nível socioeconômico, que não podem ser deixados de lado ao analisar LV 21. Porém, nem todos os fatores citados, foram explorados nesta análise.

No Tocantins, as mais altas taxas de incidência ocorrem no norte do estado. Nessa região, foram detectados clusters em que coincidem a baixa elevação e a alta taxa de incidência de LV. Tais clusters podem ser explicados pela presença do vetor da $\mathrm{LV}$, em maior quantidade, em altitudes mais baixas. Isso também foi identificado em estudos epidemiológicos de focos endêmicos de LV na Etiópia 


\section{Figura 3}

Mapas de clusters de Indicador Local de Associação Espacial (LISA - Local Indicators of Spatial Association) bivariado entre as variáveis climáticas e ambientais e a taxa de incidência de leishmaniose visceral (LV) dos municípios do Estado do Tocantins, Brasil.

3a) Elevação vs. taxa de incidência de LV

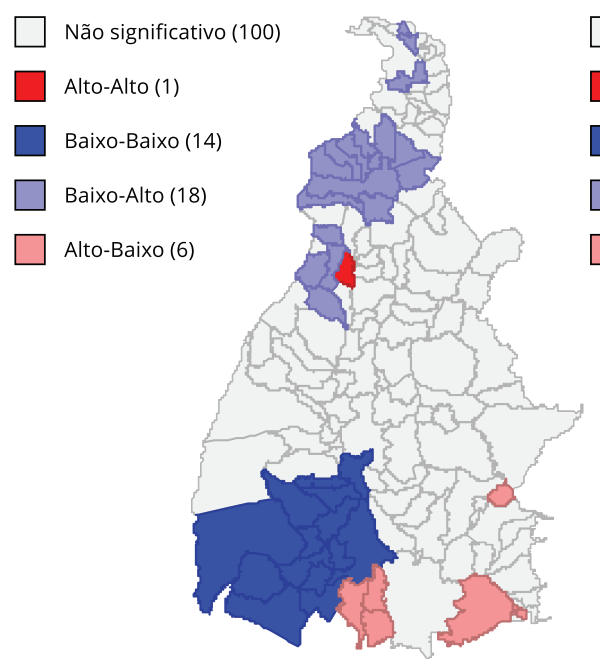

3b) Temperatura diurna vs. taxa de incidência de LV

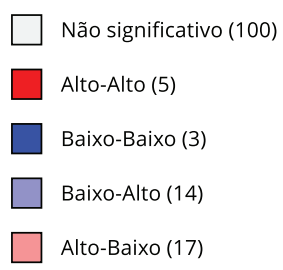

Alto-Baixo (17) 3d) EVI 2007 vs. taxa de incidência de LV

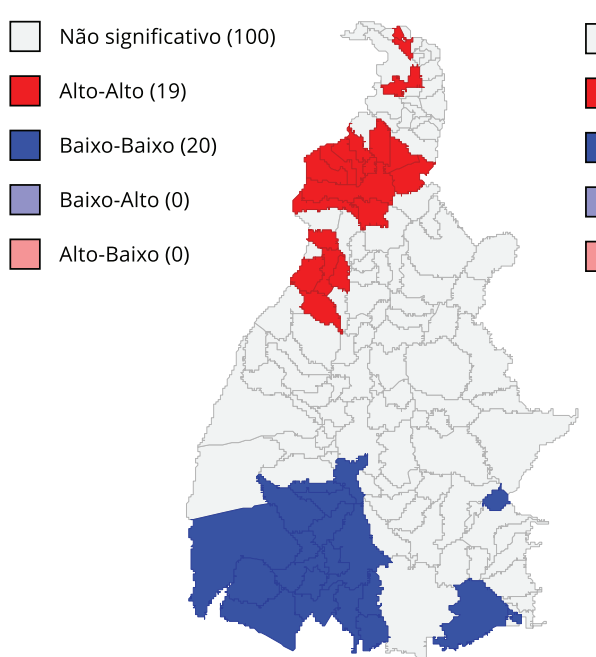

3e) EVI 2014 vs. taxa de incidência de LV

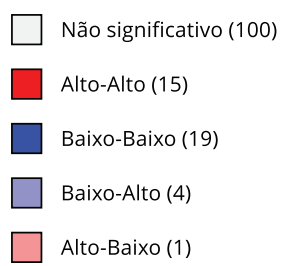

Alto-Baixo (1)

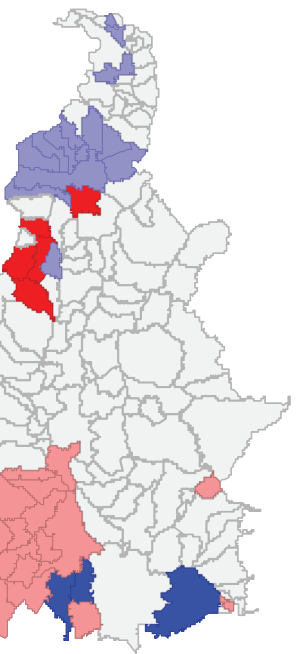

3c) Temperatura noturna vs. taxa de incidência de LV

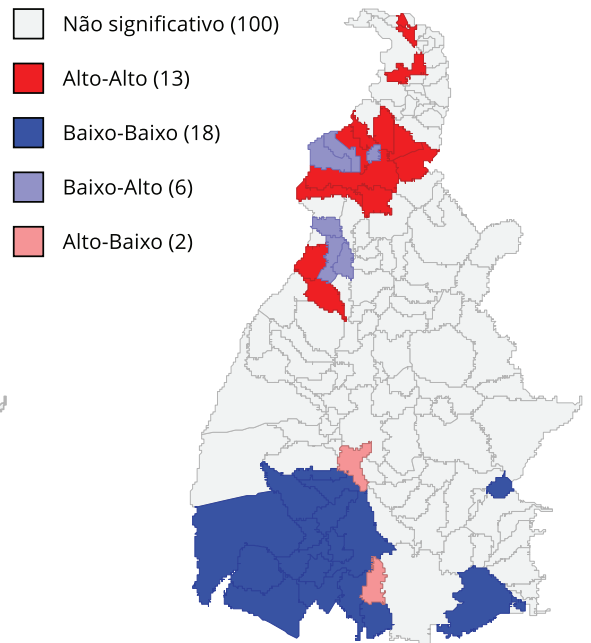

3f) Precipitação pluviométrica vs. taxa de incidência de LV

$\square$ Não significativo (100)
$\square \quad$ Alto-Alto (19)
$\square \quad$ Baixo-Baixo (18)
$\square \quad$ Baixo-Alto (0)
$\square \quad$ Alto-Baixo (2)
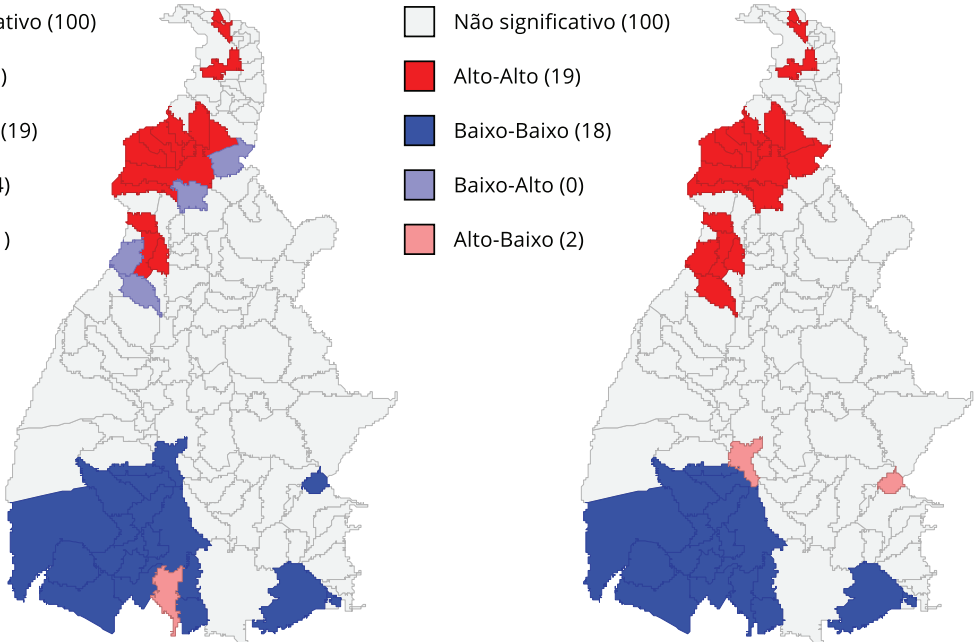

EVI: Índice de Vegetação Melhorado (Enhanced Vegetation Index).

Nota: as variáveis referem-se ao ano de 2010, com exceção do EVI de 2007 e EVI de 2014. Pseudo valor de p com 999 permutações para avaliar a significância de $1 \%$. 
(altitude abaixo de 1.794m) 22, assim como em Belo Horizonte (Minas Gerais), onde estudos identificaram mais ocorrência de casos humanos de LV e de casos caninos de LV em altitudes entre 780 $880 \mathrm{~m}$ 23; e de casos humanos em altitudes mais baixas $(<949 \mathrm{~m}){ }^{24}$, ou maior frequência do vetor em altitudes abaixo de $851 \mathrm{~m} 25$. Todavia, também foram detectados no sul do estado clusters em que ocorre baixa elevação e baixa taxa de incidência. Tal fato contradiz esses estudos e faz pensar que nas mais baixas altitudes ocorrem tanto taxas de incidência altas quanto baixas, sugerindo que a taxa de incidência pode estar sendo influenciada por outras variáveis, além da elevação. Por outro lado, as altitudes (102 a 631m) nos municípios de Tocantins são inferiores às dos estudos citados acima, em que as mais baixas altitudes citadas nos estudos coincidem com áreas mais elevadas de Tocantins.

Quanto à temperatura, parece haver um valor ideal que pode favorecer a ocorrência de mais casos. Apesar da pequena variação da temperatura nos municípios do Tocantins, foram identificados, em maior quantidade, clusters em locais com alta temperatura noturna e alta taxa de incidência, e clusters de municípios com baixa temperatura noturna e baixa taxa de incidência. Em relação a variável temperatura diurna, foram identificados clusters com baixa temperatura diurna e alta taxa de incidência, e clusters com alta temperatura diurna e baixa taxa de incidência. Assim, nos clusters de taxa de incidência alta, ocorrem temperatura noturna alta e temperatura diurna baixa. Ao que parece, não seriam nem temperaturas muito elevadas e nem tão baixas, associadas à ocorrência da LV neste estado 26 , pois assim como se cita a relação da temperatura com a reprodução do parasito, esta também influencia a atividade vetorial. A L. longipalpis apresenta tendência a ser mais ativa à noite, em temperatura de $30^{\circ} \mathrm{C} 18$, que coincide com as baixas temperaturas diurnas identificadas neste estudo. Os flebotomíneos são vulneráveis a mudanças bruscas de temperatura e, geralmente, preferem regiões nas quais ocorre baixa variação entre as temperaturas mínimas e máximas, visto que sua sobrevivência pode ser reduzida se o clima ficar muito quente e seco 16,27 e, dessa maneira, temperaturas altas diminuem também os casos de LV humana 26. Também foi observado que temperaturas mais baixas aumentam a longevidade e quantidade do flebotomíneo 28,29, o que pode aumentar a incidência de LV. Tal fato foi detectado neste estudo, pois se obteve correlação positiva entre temperatura noturna e taxa de incidência.

Em relação a variável precipitação pluviométrica, os clusters com alta precipitação e alta taxa de incidência, e clusters com baixa precipitação e baixa taxa demonstram a correlação espacial positiva entre essas variáveis. A variável precipitação pluviométrica, que se correlaciona com a umidade, favorece o desenvolvimento e reprodução do vetor e consequente aumento da incidência de LV 30,31,32. Como Tocantins apresenta características do bioma Amazônia e do cerrado, com o bioma Amazônia ocorrendo mais ao norte do estado, o regime de chuvas da floresta tropical exerce forte influência na pluviosidade e consequente umidade do estado, contribuindo para incidência da LV nessa região do estado.

Com os dados de vegetação, foram identificados clusters nos quais ocorrem alto EVI e alta taxa de incidência e também clusters com baixo EVI e baixa taxa de incidência. Isso pode estar relacionado à urbanização não planejada em direção às áreas de floresta e suas bordas. Salienta-se que 90,6\% dos casos são de residentes da área urbana no Estado do Tocantins (dados não apresentados). Talvez, a pressão por necessidade de moradia associada à falta de infraestrutura urbana possa ocasionar mais proximidade dos moradores com áreas de floresta, favorecendo o contato com o vetor e aumento na transmissão da doença 21. A proximidade de áreas com vegetação mais densa pode facilitar a transmissão fornecendo as condições para a manutenção da população do vetor da LV e do reservatório silvestre 33,34. Dessa forma, a expansão desordenada das cidades que avançam em meio ao habitat natural do vetor da LV, associada às condições climáticas e ambientais favoráveis, aproxima o contato do vetor com animais domésticos e, consequentemente, mais ocorrência da doença. Entretanto, em áreas que passaram por um processo mais antigo de urbanização, o vetor se encontra bem adaptado em ambiente urbano, sendo encontrado dentro e no peridomicílio, onde provavelmente ocorra pouca vegetação 35 .

A correlação entre a taxa de incidência de LV e as variáveis climáticas e ambientais detectada neste estudo nos permite compreender a ocorrência da LV em Tocantins. Como é um estado que se apresenta como um misto entre as regiões Norte e Centro-oeste do Brasil, cujos biomas diferenciados contribuem para a doença, há evidências de não aleatoriedade espacial na distribuição da doença com as variáveis climáticas e ambientais. No entanto, outros fatores não explorados aqui também 
podem ser responsáveis pela doença, inclusive o movimento e composição populaciona humana, assim como o modo de vida da população, a infraestrutura sanitária e as condições de vida 21,36 , que podem empurrar o homem para mais próximo do contato com o vetor, já que a ocorrência da doença em uma determinada área depende basicamente da presença do vetor suscetível e de um hospedeiro/ reservatório igualmente suscetível 37 .

Algumas limitações deste estudo são imanentes aos dados, uma vez que se trabalhou com a média das variáveis ambientais, unificando tempo e espaço. Ou seja, a média se refere a cada município, de dados registrados pelos satélites, em diferentes pontos do tempo, os quais foram analisados como média anual. Portanto, podem não corresponder exatamente a uma correlação com a ocorrência da doença em um ponto exato. Todavia, há de se ter em conta que a correlação identificada entre taxa de incidência e variáveis ambientais apresenta um padrão espacial que não pode ser considerado aleatório.

Por outro lado, a exatidão das estimativas ambientais pode ser comprometida pela precisão dos satélites, como no SRTM que possui precisão de $90 \mathrm{~m} 38$, e pela distribuição das estações de captação no território. Alguns eventos também podem comprometer a captura dos dados, como precipitações em áreas isoladas, que não corresponde à área total do município, inclinação do terreno 39 , que pode gerar imprecisão dos dados de elevação, e condições atmosféricas, que interferem na captação dos dados de EVI. Entretanto, o EVI é um índice desenvolvido para minimizar os efeitos de potenciais interferências, e apresenta uma linearidade aos parâmetros biofísicos em diversas condições de vegetação ${ }^{12}$. Além disso, a baixa variabilidade dos dados pode ser atribuída ao pequeno tamanho da área em estudo, mesmo que se considere que o Estado de Tocantins apresente ambos os biomas, do cerrado e da floresta amazônica.

A taxa de LV utilizada neste estudo foi calculada mediante os casos notificados ao SINAN, portanto as subnotificações e os casos não diagnosticados da doença não foram investigados, o que pode configurar uma subestimação da taxa. Todavia, há de se considerar que, mesmo com a possibilidade de subenumeração de casos, tanto a taxa de incidência em Tocantins é elevada, quanto as relações identificadas que mostram que a doença possui um padrão espacial a ser levado em conta, em combinação com as condições ambientais.

\section{Colaboradores}

L. L. Reis e M. J. F. Gonçalves contribuíram com a análise e interpretação dos dados, elaboração do manuscrito e revisão crítica do conteúdo e participaram da aprovação da versão final do manuscrito. A. A. S. Balieiro e F. R. Fonseca contribuíram com a análise e interpretação dos dados e participaram da aprovação da versão final do manuscrito.

\section{Agradecimentos}

Ao Ministério da Saúde pela cessão dos dados de leishmaniose visceral no Brasil. 


\section{Referências}

1. Reis LL, Balieiro AAS, Fonseca FR, Gonçalves MJF. Changes in the epidemiology of visceral leishmaniasis in Brazil from 2001 to 2014. Rev Soc Bras Med Trop 2017; 50:638-45.

2. Afonso MMS, Chaves SAM, Magalhães MAFM, Gracie R, Azevedo C, Carvalho BM, et al. Ecoepidemiology of American visceral leishmaniasis in Tocantins State, Brazil: factors associated with the occurrence and spreading of the vector Lutzomyia (Lutzomyia) longipalpis (Lutz \& Neiva, 1912) (Diptera: Psychodidae: Phlebotominae). In: Claborn D, editor. The epidemiology and ecology of leishmaniasis. Rijeka: InTech; 2017. p. 91-115.

3. Silva LAGC. Biomas presentes no Tocantins. Biblioteca Digital da Câmara dos Deputados. http://bd.camara.gov.br/bd/handle/bdcama$\mathrm{ra} / 1424$ (acessado em 08/Jul/2017).

4. Ministério da Saúde. Portaria no 104, de 25 de janeiro de 2011. Define as terminologias adotadas em legislação nacional, conforme o disposto no Regulamento Sanitário Internacional 2005 (RSI 2005), a relação de doenças, agravos e eventos em saúde pública de notificação compulsória em todo o território nacional e estabelece fluxo, critérios, responsabilidades e atribuições aos profissionais e serviços de saúde. Diário Oficial da União 2011; 26 jan.

5. Conselho Nacional de Saúde. Resolução no 510, de 07 de abril de 2016. Diário Oficial da União 2016; 24 mai.

6. Organización Panamericana de la Salud. Leishmaniasis - informe epidemiológico de las Américas. Informe de Leishmaniasis 2016; (4):3-7.

7. Instituto Brasileiro de Geografia e Estatística. Indicadores sociais mínimos - conceitos. https://ww2.ibge.gov.br/home/estatistica/popu lacao/condicaodevida/indicadoresminimos/ conceitos.shtm (acessado em 08/Mai/2018).

8. Farr TG, Rosen PA, Caro E, Crippen R, Duren R, Hensley S, et al. The Shuttle Radar Topography Mission. Rev Geophys 2007; 45(RG2004):1-33.

9. Funk CC, Peterson PJ, Landsfeld MF, Pedreros DH, Verdin JP, Rowland JD, et al. A quasi-global precipitation time series for drought monitoring. Reston: U.S. Geological Survey; 2014. (Data Series, 832).

10. Saha S, Moorthi S, Wu X, Wang J, Nadiga S, Tripp P, et al. The NCEP Climate Forecast System version 2. J Clim 2014; 27:2185-208.

11. Wan Z, Hook S, Hulley G. MOD11A2: MODIS/Terra Land Surface Temperature/Emissivity 8-Day L3 Global 1km SIN Grid V006. https://lpdaac.usgs.gov/node/820 (acessado em 14/Mar/2015).

12. Huete A, Didan K, Miura T, Rodriguez EP, Gao $X$, Ferreira LG. Overview of the radiometric and biophysical performance of the MODIS vegetation indices. Remote Sens Environ 2002; 83:195-213.
13. Câmara G, Carvalho MS, Cruz OG, Correia VRM. Análise espacial de áreas. In: Druck S, Carvalho MS, Câmara G, Monteiro AMV, organizadores. Análise espacial de dados geográficos. v. 1. Brasília: Empresa Brasileira de Pesquisa Agropecuária; 2004. p. 157-209.

14. Anselin L, Syabri I, Smirnov O. Visualizing multivariate spatial correlation with dynamically linked windows. In: Anselin L, Rey S, editors. New tools for spatial data analysis: proceedings of the specialist meeting [CD-ROM] Santa Barbara: Center for Spatially Integrated Social Science, University of California; 2002.

15. Secretaria de Vigilância em Saúde, Ministério da Saúde. Guia de vigilância em saúde. Brasília: Ministério da Saúde; 2014.

16. Abrantes P, Silveira H. Alterações climáticas na Europa: efeito nas doenças parasitárias humanas. Rev Port Saúde Pública 2009; 27:71-86.

17. Galati EAB, Camara TNL, Natal D, Chiaravalloti-Neto F. Mudanças climáticas e saúde urbana. Rev USP 2015; 107:79-90.

18. Rivas GB, Souza NA, Peixoto AA, Bruno RV. Effects of temperature and photoperiod on daily activity rhythms of Lutzomyia longipalpis (Diptera: Psychodidae). Parasit Vectors 2014; $7: 278$.

19. Serafim TD, Coutinho-Abreu IV, Oliveira F, Meneses C, Kamhawi S, Valenzuela JG. Sequential blood meals promote Leishmania replication and reverse metacyclogenesis augmenting vector infectivity. Nat Microbiol 2018; 3:548-55

20. Antero R. Urbanização pela migração em Araguaína (TO). Caminhos de Geografia 2016; 17:228-43

21. Toledo RCS, Almeida AS, Chaves MAS, Sabroza PC, Toledo LM, Caldas JP. Vulnerabilidade à transmissão da leishmaniose visceral humana em área urbana brasileira. Rev Saúde Pública $2017 ; 51: 49$.

22. Tsegaw T, Gadisa E, Seid A, Abera A, Teshome A, Mulugeta A, et al. Identification of environmental parameters and risk mapping of visceral leishmaniasis in Ethiopia by using geographical information systems and a statistical approach. Geospat Health 2013; 7:299-308

23. Margonari C, Freitas CR, Ribeiro RC, Moura ACA, Timbó M, Gripp AH, et al. Epidemiology of visceral leishmaniasis through spatial analysis, in Belo Horizonte municipality, state of Minas Gerais, Brazil. Mem Inst Oswaldo Cruz 2006; 101:31-8

24. Araújo VEM, Pinheiro LC, Almeida MCM, Menezes FC, Morais MHF, Reis IA, et al. Relative risk of visceral leishmaniasis in Brazil: a spatial analysis in urban area. PLoS Negl Trop Dis 2013; 7:e2540. 
25. Saraiva L, Andrade Filho JD, Falcão AL, Carvalho DAA, Souza CM, Freitas CR, et al. Phlebotominae fauna (Diptera: Psychodidae) in an urban district of Belo Horizonte, Brazil, endemic for visceral leishmaniasis: characterization of favored locations as determined by spatial analysis. Acta Trop 2011; 117:137-45.

26. Oliveira IBB, Batista HL, Peluzio JM, Pfrimer IAH, Rodrigues FM, Carmo Filho JR. Epidemiological and environmental aspects of visceral leishmaniasis in children under 15 years of age between 2007 and 2012 in the city of Araguaína, State of Tocantins, Brazil. Rev Soc Bras Med Trop 2014; 47:476-82.

27. Rajesh K, Sanjay K. Change in global climate and prevalence of visceral leishmaniasis. International Journal of Scientific and Research Publications 2013; 3:1-2.

28. Guzmán H, Tesh RB. Effects of temperature and diet on the growth and longevity of phlebotomine sand flies (Diptera: Psychodidae). Biomédica (Bogotá) 2000; 20:190-9.

29. Tonelli GB, Tanure A, Rêgo FD, Carvalho GML, Simões TC, Andrade Filho JD. Aspects of the ecology of phlebotomine sandflies (Diptera: Psychodidae) in the Private Natural Heritage Reserve Sanctuary Caraça. PLoS One 2017; 12:e0178628

30. Lara-Silva FO, Michalsky EM, Fortes-Dias CL, Fiuza VOP, Pessanha JEM, Regina-Silva S, et al. Epidemiological aspects of vector, parasite, and domestic reservoir in areas of recent transmission and no reported human cases of visceral leishmaniasis in Brazil. Acta Trop 2015; 148:128-36.

31. Lima ID, Lima ALM, Mendes-Aguiar CO, Coutinho JFV, Wilson ME, Pearson RD, et al. Changing demographics of visceral leishmaniasis in northeast Brazil: lessons for the future. PLoS One 2018; 12:e006164.

32. Galvis-Ovallos F, Casanova C, Sevá ADP, Galati EAB. Ecological parameters of the (S)9-methylgermacrene-B population of the $\mathrm{Lu}$ tzomyia longipalpis complex in a visceral leishmaniasis area in São Paulo state, Brazil. Parasit Vectors 2017; 10:269.
33. Costa AP, Costa FB, Soares HS, Ramirez DG, Araújo AC, Ferreira JIG, et al. Environmental factors and ecosystems associated with canine visceral leishmaniasis in Northeastern Brazil. Vector Borne Zoonotic Dis 2015; 15:765-74.

34. Cerbino Neto J, Werneck GL, Costa CHN. Factors associated with the incidence of urban visceral leishmaniasis: an ecological study in Teresina, Piauí State, Brazil. Cad Saúde Pública 2009; 25:1543-51.

35. Silva RA, Santos FKM, Sousa LC, Rangel EF, Bevilaqua CML. Ecology of Lutzomyia longipalpis and Lutzomyia migonei in an endemic area for visceral leishmaniasis. Rev Bras Parasitol Vet 2014; 23:320-7.

36. Vasconcelos Filho JM, Ribeiro Soares B. Os conflitos e as contradições na aquisição da moradia social nos enclaves de pobreza urbana no Brasil: uma análise sobre a cidade de Araguaína-TO. Revista Geográfica de América Central 2011; 2:1-14.

37. Gontijo C, Melo M. Leishmaniose visceral no Brasil: quadro atual, desafios e perspectivas. Rev Bras Epidemiol 2004; 7:338-49.

38. Gorokhovich Y, Voustianiouk A. Accuracy assessment of the processed SRTM-based elevation data by CGIAR using field data from USA and Thailand and its relation to the terrain characteristics. Remote Sens Environ 2006; 104:409-15.

39. Mukherjee S, Joshi PK, Mukherjee S, Ghosh A, Garg RD, Mukhopadhyay A. Evaluation of vertical accuracy of open source Digital Elevation Model (DEM). Int J Appl Earth Obs Geo$\inf 2013 ; 21: 205-17$ 


\section{Abstract}

Visceral leishmaniasis (VL) may be influenced by climate and environmental factors. Tocantins is the state with the highest incidence in Brazil, contributing to the increase in the number of cases in the North macro-region. This study seeks to identify the correlation between VL incidence rates and climate and environmental factors in the municipalities of the State of Tocantins between 2007 and 2014. Correlations between crude VL incidence rates and the variables elevation, precipitation, Enhanced Vegetation Index (EVI) and temperature were tested using the Global and Local Moran Indexes, while also identifying clusters of $V L$ incidence. We tested a variance analysis for linear trend between variable means in each rate category. Results show an increase in incidence rates with an increase in values of annual precipitation, humidity, EVI and nighttime temperature; and an inverse relationship with elevation and daytime temperature. The clusters we identified show that climate and environmental variables are related to $V L$ incidence. Other studies are needed that adjust for individual-related factors in order to gauge how much the environment, or human influence over it, impact the disease.

Temperature; Pluviometry; Altitude; Humidity; Visceral Leishmaniasis

\section{Resumen}

La leishmaniasis visceral ( $L V)$ puede estar influenciada por factores climáticos y ambientales. Tocantins es el estado con más incidencia en Brasil, contribuyendo al aumento de casos en la macrorregión Norte. Este estudio tiene como objetivo identificar la correlación entre la tasa de incidencia de LV y los factores climáticos y ambientales, en municipios del Estado de Tocantins de 2007 a 2014. Las correlaciones entre la tasa de incidencia bruta de $L V$ y las variables elevación, precipitación, Índice Mejorado de Vegetación (EVI - Enhanced Vegetation Index) y temperatura se comprobaron mediante los Índices de Moran Global y Local, identificando también clústeres de incidencia de LV. El análisis de varianza para la tendencia lineal se probó entre las medias de las variables en cada categoría de la tasa. Los resultados apuntan un aumento en las tasas de incidencia, a medida que aumentan los valores de precipitación anual, humedad, EVI y temperatura nocturna; y una relación inversa respecto a la elevación y temperatura diurna. Los clústeres identificados evidencian qué variables climáticas $y$ ambientales se relacionan con la incidencia de $L V$, para lo que deben existir otros estudios que ajusten los factores asociados a los sujetos, con el fin de dimensionar lo mucho que el ambiente o la influencia del hombre impacta en la enfermedad.

Temperatura Ambiental; Pluviometría; Altitud; Humedad; Leishmaniasis Visceral

Recebido em 13/Mar/2018

Versão final reapresentada em 10/Set/2018

Aprovado em 01/Out/2018 